\title{
New perspectives on the nutritional factors influencing growth rate of Candida albicans in diabetics. An in vitro study
}

\author{
Adrian Man', Cristina Nicoleta Ciurea², Dan Pasaroiu², Ana-loana Savin², \\ Felicia Toma ${ }^{1,4}$, Floredana Sular ${ }^{3,4}$, Luigi Santacroce ${ }^{5 /+}$, Anca Mare ${ }^{1}$ \\ ${ }^{1}$ University of Medicine and Pharmacy, Department of Microbiology, Tîrgu Mures, Romania \\ ${ }^{2}$ University of Medicine and Pharmacy, Tîrgu Mures, Romania \\ ${ }^{3}$ University of Medicine and Pharmacy, Department of Laboratory Medicine, Tîrgu Mures, Romania \\ ${ }^{4}$ Emergency Clinical County Hospital, Tîrgu Mures, Romania \\ ${ }^{5}$ University of Bari Aldo Moro, Policlinico University Hospital, Ionian Department and Microbiology and Virology Service, Bari, Italy
}

BACKGROUND The link between Candida albicans and diabetes mellitus is well-acknowledged, but incompletely elucidated.

OBJECTIVES The purpose of this study is to assess the growth rate of C. albicans (CA) in the presence of different concentrations of glucose and fructose, two of the main pathophysiologic and nutritionally relevant sugars in diabetic patients, in order to obtain a better understanding of the nutrient acquisition strategy and its possible relation to the hyperglycemic status of diabetic patients.

METHODS The effects of different concentrations of glucose and fructose $(1000 \mathrm{mg} \%, 500 \mathrm{mg} \%, 250 \mathrm{mg} \%$ and $100 \mathrm{mg} \% \mathrm{w} / \mathrm{v})$ on the growth rate of CA have been studied by flow-cytometry.

FINDINGS We found that glucose concentration is directly related to CA growth, which may be linked to the frequent yeast infections that occur in non-controlled diabetic patients; we also show that fructose inhibits CA growth rate.

MAIN CONCLUSIONS As a consequence of our hypothesis, the study demonstrates that fructose-containing food may prevent the development of candidiasis, at least in oral sites.

Key words: Candida albicans - diabetes - yeast infections - fructose - flow-cytometry - glycemic control

It is well known that diabetes mellitus is one of the most prevalent chronic conditions throughout the world, with an estimation of 56 million cases, a prevalence of 8.5\% in Europe in 2013 and rising (Tamayo et al. 2014). The high morbidity is mostly related to chronic macrovascular and microvascular complications such as nephropathy, neuropathy, retinopathy, heart complications, related obesity, but also infectious complications, due to the hyperglycemic environment (Casqueiro et al. 2012, Tamayo et al. 2014, Gregg et al. 2016). Yeast infections in diabetic patients are common, candidiasis being described as part of the major infectious pathology: oral and esophageal candidiasis, pyelonephritis or cystitis (Casqueiro et al. 2012). In addition, recent reviews have focused on the relationships between oral microbial flora, oral hygiene and glycemic control in diabetics (Santacroce et al. 2010, Monea et al. 2017).

Although the link between Candida albicans (CA) and diabetes mellitus is well-recognised, it is still incompletely elucidated (Soysa et al. 2006). Oral candidiasis is frequently caused by albicans species and presents many clinical forms (pseudomembranous candidiasis, Candidarelated leukoplakia, angular cheilitis, glossitis or stomatitis). Candida spp. is frequently found in patients with poor glycemic control, and it was already shown that increased

doi: 10.1590/0074-02760170098

+ Corresponding author: luigi.santacroce@uniba.it

Received 11 March 2017

Accepted 15 April 2017 salivary glucose levels lead to increased oral Candida carriage (Al-Maskari et al. 2011, Kumar et al. 2014).

This in vitro pilot study tries to further define the links between the high level of glucose present in blood and secretions (including saliva) of diabetic patients, and the development of candidiasis in these categories of patients, by combining our findings with references from the literature. The purpose of this study is to assess the growth rate of $\mathrm{CA}$ in the presence of different concentrations of glucose and fructose, two of the main physiopathologically and nutritionally relevant sugars in diabetic patients, in order to obtain a better understanding of the nutrient acquisition strategy and its possible relation to the hyperglycemic status of diabetic patients. The in vitro study of Candida behavior in the presence of glucose is an attempt to recreate one of the conditions this fungus meets in a diabetic organism. Furthermore, we studied CA behavior in fructose environment, in order to obtain a better understanding of the nutrient acquisition strategy and implication of this glucose substituent from diabetic food products upon the Candida replication rate.

We hypothesize that fructose, a sugar that is generally metabolised slower than glucose, will negatively affect CA growth. Standard plate count (SPC) and optical density (OD) measurements are commonly used methods for determining microbial growth (Biesta-Peters et al. 2010). Our previous experiments (unpublished data) showed that SPC is not a reliable method for following CA growth rate, because of high variation between replicates. In order to achieve more consistent results, we assessed the yeast cell population dynamics using the Apogee A50-Micro 
flow-cytometer (Apogee Flow Systems, UK), specially designed for counting small biological particles, due to its better light scatter performance (Foladori et al. 2010).

\section{MATERIALS AND METHODS}

A 0.5 McFarland suspension (approximately 1-3 x $10^{6}$ yeast cells $/ \mathrm{mL}$ ) was created from fresh, 24-hour culture of C. albicans (ATCC 10231) in saline solution. The strain is part of the microbe collection of the Microbiology Department of the University of Medicine and Pharmacy of Tirgu Mures, Romania. After thawing an aliquot from $-80^{\circ} \mathrm{C}$ freezer, the strain was subcultured twice on Sabouraud dextrose agar (Becton, Dickinson and Company, Sparks, USA) at $35^{\circ} \mathrm{C}, 24 \mathrm{~h}$ before each experiment. Ten microliters of the $0.5 \mathrm{McF}$ arland suspension were inoculated in $990 \mu \mathrm{L}$ of sterile nutrient broth (Oxoid, UK). Pure glucose or fructose powder was added in order to obtain stock solutions of $1000 \mathrm{mg} \% \mathrm{w} / \mathrm{v}$. Lower concentrations of sugars $(500 \mathrm{mg} \%$, $250 \mathrm{mg} \%$ and $100 \mathrm{mg} \% \mathrm{w} / \mathrm{v}$, respectively) were prepared by diluting appropriate volumes of the stock solution in nutrient broth. One sample tube with no added sugar served as growth control. Each experiment was made in triplicate. All the samples were incubated in a shaking mixer at $37^{\circ} \mathrm{C}$, in normal atmosphere, for a total of $9 \mathrm{~h}$. At starting time ( $\mathrm{HO})$ and every $3 \mathrm{~h}(\mathrm{H} 1=3 \mathrm{~h} ; \mathrm{H} 2=6 \mathrm{~h} ; \mathrm{H} 3=9 \mathrm{~h})$, the number of yeast cells was assessed by flow-cytometry using Apogee A50-Micro flow-cytometer (Apogee Flow Systems, UK), by counting the number of events in $10 \mu \mathrm{L}$ of each fungal suspension in a gating area on LALS/SALS scatterplot cytogram that corresponds to the yeast cells population. The obtained values were adjusted to obtain the number of colony forming units $/ \mathrm{mL}(\mathrm{CFU} / \mathrm{mL})$. Each sample was shortly but thoroughly vortexed three times before aspiration, in order to disperse and evenly spread the yeast cells. For a better accuracy, the tests were conducted in triplicate (three tubes for each sample). Because of the complexity of time requirements of this experiment, the glucose and fructose were assessed in two different days, without major variation regarding the environmental and processing conditions.

The CFU $/ \mathrm{mL}$ values for all samples were normalised against the values obtained at $\mathrm{H} 0$ and expressed as $\Delta$-Index, which compensated the errors regarding inocu- lum and sample preparation (the CFU number at starting point $\mathrm{H} 0$ ). The second normalisation was performed between the $\Delta$-Index of the samples and $\Delta$-Index of the controls $(\mathrm{CFU} / \mathrm{mL}$ values of the samples with no added sugars), and expressed as $\Delta \Delta$-Index, which compensated the growth variation due to external or other non-identifiable factors, thus making it a better growth marker. A $\Delta \Delta$-Index value higher than one is equivalent to growth enhancement, while a value less than one is equivalent to growth inhibition, compared to control.

Complementary to the normalised indices, we assessed the growth rate per hour (r) and the generation time $(\mathrm{g})$ in minutes, which were calculated using the absolute numbers of $\mathrm{CFU} / \mathrm{mL}$ read at $\mathrm{H} 3$ compared to $\mathrm{H} 0$, according to the following formula:

$$
\begin{gathered}
r=\operatorname{In} \frac{C F U / m L \text { for } T 1-C F U / m L \text { for } T 0}{\text { no. hours for } T 1} \\
g=\frac{\operatorname{In}(2)}{r} * 60
\end{gathered}
$$

Explanation: $r$ - growth rate; $T 1$ - time of sample reading (in hours); T0 - initial time (0 hours reading)

All the statistical evaluations were performed in spreadsheet software and GraphPad InStat3. The significance threshold was considered for a p-value of less than 0.05 .

\section{RESULTS}

Presence of glucose and fructose presented opposite effects on the growth rate of CA. Overall, glucose had a stimulatory effect while fructose an inhibitory effect on the growth of CA after $9 \mathrm{~h}$ of incubation (Fig. 1). In absolute values, all samples presented an exponential, significant growth $(p<0.005)$. The first $3 \mathrm{~h}$ were part of the lag phase, when the number of CFU was constant or decreasing. At $\mathrm{H} 2$, after $6 \mathrm{~h}$ of incubation, the yeast population was less than double in the case of fructose ( $\Delta$-Index between 1.14 and 1.98) or up to 12 folds increased in the case of glucose ( $\Delta$-Index between 8.86 and 12.11).

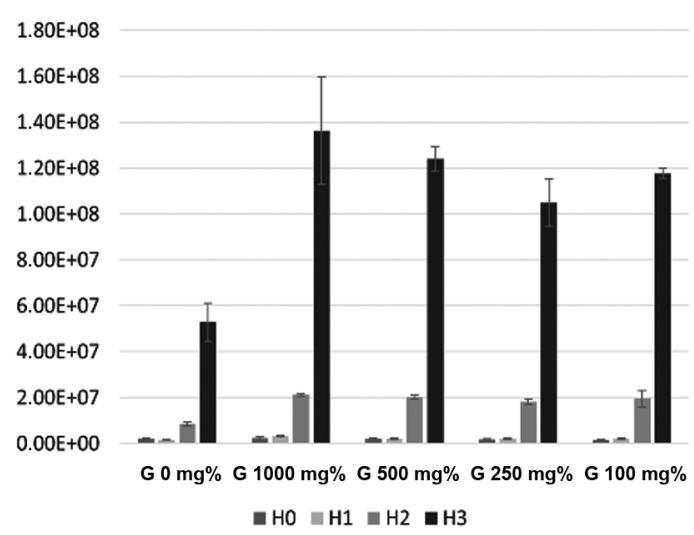

A

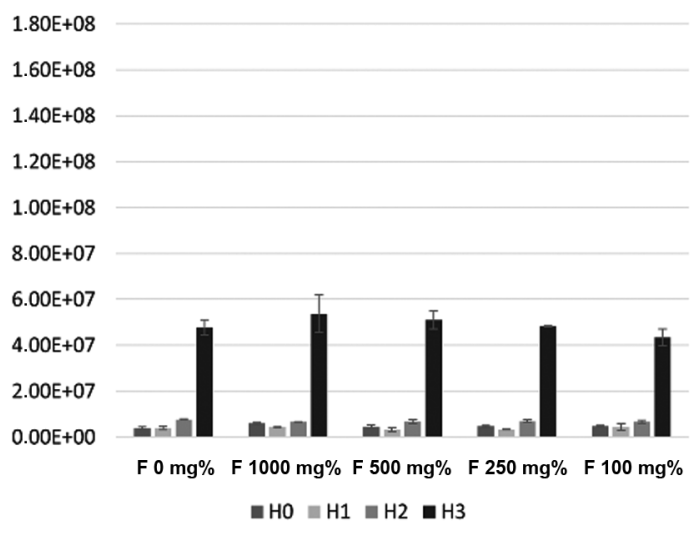

B

Fig. 1: differences in the number of $\mathrm{CFU} / \mathrm{mL}$ (mean values) after $9 \mathrm{~h}$ of incubation in presence of glucose (A) and fructose (B). 


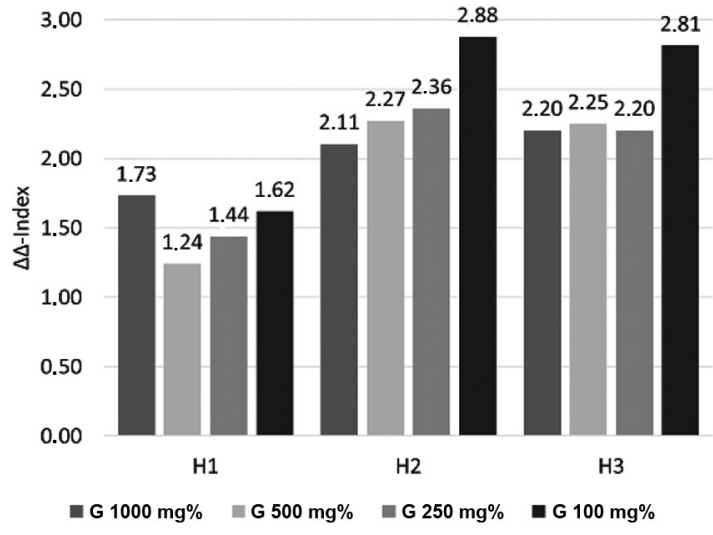

A

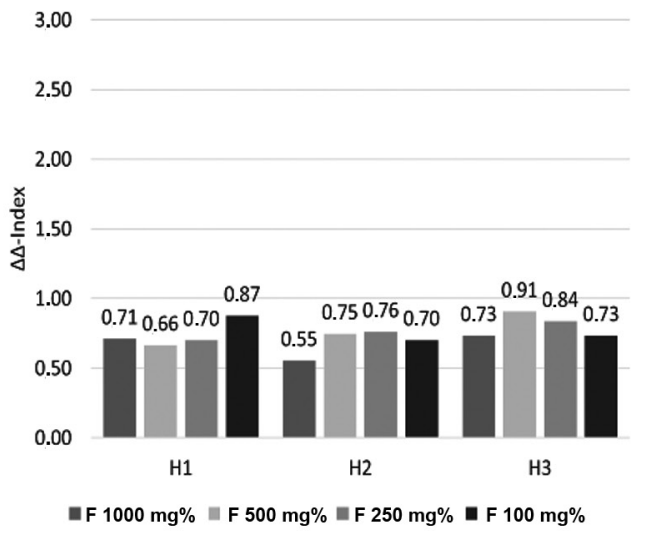

B

Fig. 2: normalised indices for Candida albicans growth in presence of different concentrations of glucose (A) or fructose (B).

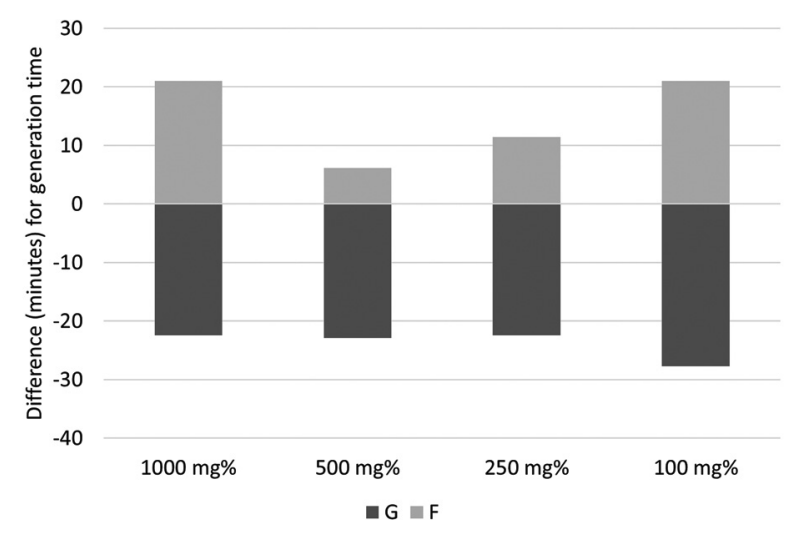

Fig. 3: alteration of generation time in presence of glucose (a) and fructose (b).

The high concentration of glucose (1000 mg\%) provided a boost effect on the growth rate of CA as read after $3 \mathrm{~h}$ of incubation (H1) but this effect almost leveled during the last $6 \mathrm{~h}$. Instead, glucose $100 \mathrm{mg} \%$ presented a milder but sustained effect after the $9 \mathrm{~h}$ of incubation, this concentration being the most potent simulator of CA growth rate. Fructose presented an inhibitory effect on CA growth, regardless of its concentration (Fig. 2). The growth rate is directly related to the generation time. Glucose presence decreased the generation time with more than $20 \mathrm{~min}$, while fructose presence increased it with approximatively $15 \mathrm{~min}$. In absolute values, the growth rate/hour ratio was higher in glucose presence $(\mathrm{r}=0.45-0.48)$ than in fructose presence $(\mathrm{r}=0.25-0.27)$. Generation time varied between 87-92 min for glucose and 154-166 min for fructose (Fig. 3).

The flow-cytometry showed a yeast population that was visible at $\mathrm{H} 0$, with no significantly different morphology at $\mathrm{H} 1$, which started to be better defined but still largely spread at $\mathrm{H} 2$, and condensed and well defined after $9 \mathrm{~h}$ of incubation (H3). A second population made of cell debris and probably particles from the culture medium was noticed in all samples, and ignored. These trends were noticed for both sugars. For glucose, even in the concentration that least stimulated the growth rate of $\mathrm{CA}$, the cell population was visibly enhanced at $\mathrm{H} 2$ and $\mathrm{H} 3$, compared with the corresponding scatter plots of fructose. The small yeast cells (young CA cells) are visible in presence of glucose in the lower-left corner of the gating area at $\mathrm{H} 2$ (Fig. 4).

\section{DISCUSSION}

Yeasts and fungi are frequently reported as responsible of severe conditions affecting the cervical and facial areas of patients suffering from immune disorders and diabetics (Giudice et al. 2016), and Candida spp. are the most common opportunistic fungal pathogens, CA accounting for more than $50 \%$ of the cases (Minea et al. 2015).

The candidiasis can be easily recognised, even if other clinical pictures could simulate the same aspect, as some syndromic conditions (Marrelli et al. 2012), some oncological lesions (Santacroce et al. 2005, Petrovan et al. 2017) or aggressive bacterial infections in immunocompromised patients or as results of infections from foreign bodies (Inchingolo et al. 2011).

The pathogenicity mechanisms of CA are limited compared to those present in bacteria, making this yeast a commensal and opportunistic pathogen that is present in the oral cavity in about $75 \%$ of the population, and active in case of host-related disequilibrium such as bacterial disturbance, immune disorders or local pathologies (Jenkinson \& Douglas 2002). Hyphal forms, with high invasiveness, blastospores with high dissemination capacity, adhesins, invasins, biofilm formation, environmental factors sensing and metabolic adaptation are part of the pathogenicity factors in CA (Mayer et al. 2013). The metabolic adaptation can have a key role in diabetic patients where glucose levels are higher than in non-diabetic populations, with implication in local or disseminated candidiasis.

Sugars represent potential nutritional sources for yeasts, at least in vitro; glucose, fructose and galactose 


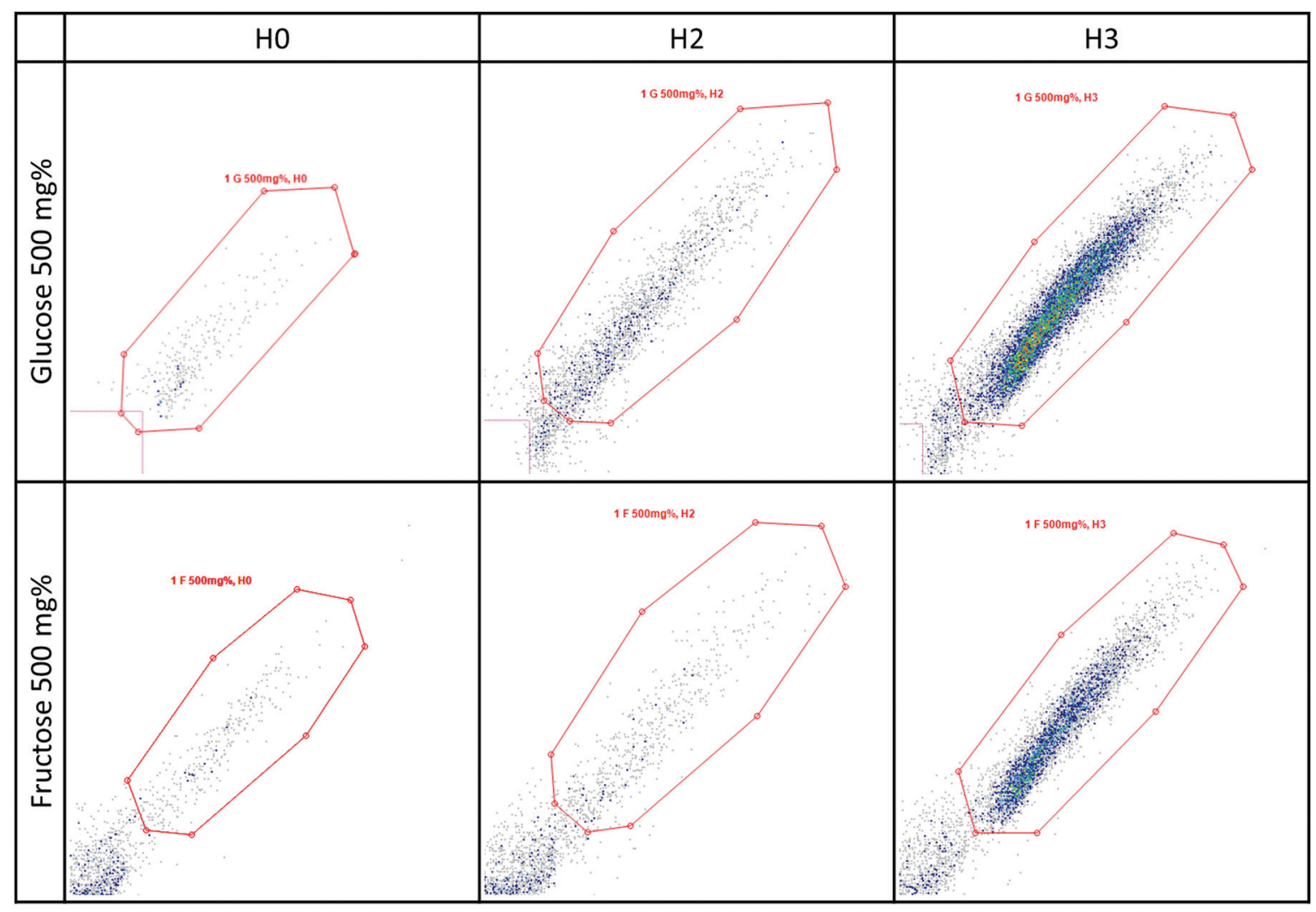

Fig. 4: images of flow-cytometry assay: Candida albicans growth in presence of glucose and fructose $500 \mathrm{mg} \%$ at three time-points.

are physiologically relevant in humans. Nevertheless, these sugars seem not to be the single nutrient sources, as lipids, proteins and amino acids are valuable alternative sources, especially in tissues with low glucose concentration (Mayer et al. 2013). Glucose and fructose enhance CA virulence by stimulating the transition from blastospores to hyphae in vivo (Sabina \& Brown 2009). Glucose concentration is higher but variable in the oral cavity (due to food habits and oral hygiene) than in blood and it seems not to have a primordial role for the burden of Candida in oral mucosa, but to enhance the burden of Candida in systemic infections (Ene et al. 2012). Nevertheless, in diabetic patients, unlike in non-diabetic ones, permeability of the basement membrane of salivary glands is affected, leading to glucose "leakage" into the saliva (Kumar et al. 2014). This explains why diabetic patients present high rates of oral candidiasis.

The growth boost in presence of $1000 \mathrm{mg} \%$ glucose was noticed in the first $3 \mathrm{~h}$ of incubation, which partly correspond to the lag phase. This can be explained by the nutritional source abundance, which provided acute energy for CA cells, but had a less favorable osmotic effect in time. Instead, a more convenient concentration of glucose $(100 \mathrm{mg} \%$ ) provided a better environment for long-time CA growth. We did not assess the effect of lower concentrations of glucose on CA growth, but we can suspect that it may similarly stimulate it. This hypothesis is supported by several studies showing that in non-controlled diabetic patients, where the salivary glucose level is higher than $12-13 \mathrm{mg} / \mathrm{dL}$, oral Candida carriage is more common (Kumar et al. 2014, Satish et al. 2014). Other studies identified patients with salivary glucose levels of $25-30 \mathrm{mg} / \mathrm{dL}$ that also correlated with high Candida carriage (Balan et al. 2015). Moreover, the salivary glucose level remains high for up to $2 \mathrm{~h}$ after food and/or sugar intake and the recovery rate to normal levels is slower in diabetic than in non-diabetic subjects (Jurysta et al. 2009). The food type also plays an important role in sugar clearance rate (Nirmala et al. 2016), thus high levels of salivary glucose are indeed achievable and may favor Candida colonisation.

Previous studies showed that fructose is metabolised more slowly than glucose by CA (Vidotto et al. 1996) and our results regarding the growth rate in presence of fructose support this. Moreover, our results showed that fructose inhibits the replication rate in CA. This may not be true in other osmotolerant and fructophilic species of Candida, which present complex sugar transport systems, including fructose-specific ones (Lee et al. 2014). In these cases, growth in presence of fructose may have a completely different trend compared to that of C. albicans, as in the latter there is either a metabolic interaction between fructose and the sugar metabolism or a complete lack of fructose transportation systems. This hypothesis needs further studies in order to establish the fructose metabolism within $C$. albicans. Given the fact that fructose improves long-term glycemic control (Cozma et al. 2012), thus being frequently used as a common sweetener 
by diabetic patients, it may also provide a certain degree of protection against CA infections. On this hypotesis, we have to mention a potential drawback of excessive fructose consumption, as described by DiNicolantonio et al. (2015): Added fructose in particular (eg., as a constituent of added sucrose or as the main component of highfructose sweeteners) may pose the greatest problem for incident diabetes, diabetes-related metabolic abnormalities, and cardiovascular risk, as the isocaloric exchange of other sugars with fructose leads to increased insulin resistance and glucose levels (Tappy \& Lê 2015).

We know that the risk of infection with Candida reported for the patients with diabetes mellitus has a multifactorial cause. In addition to the diabetic state, high sugar intake, bad eating habits or poor oral hygiene are factors that contribute to high levels of salivary glucose, and are favoring factors for oral candidiasis. Nevertheless, in this context, our study provides further proof regarding the direct link between glucose concentration and candidiasis.

In addition, our results provide information about the fungal fitness abilities in a fructose-rich environment, which raises the question whether fructose diet is a protective factor against oral candidiasis. Fructose intake, as part of diet food (sugar replacement agent) in diabetes, may play a protective role against oral candidiasis in these patients. PubMed search for the terms ("candida/ metabolism") AND "fructose" revealed 40 results, all of them referring to other Candida species than albicans. This pilot study brings new information on C. albicans metabolism related to fructose and to fructose intake that is especially met in diabetic patients. Previous studies showed that diabetes can be conveniently induced in rats (Scridon et al. 2015), so these experimental models will be the target of future in vivo experiments regarding the growth rate and infectious potential of CA. Having such consideration in mind, this study is aimed to highlight the importance of the proper clinical approach to the diabetic patients, also from the point of view of the oral care: a deep knowledge of the oral condition is important to reduce the systemic complications of some pathogenetic mechanisms (Santacroce et al. 2010).

In conclusion, in-vitro analysis of CA growth rate by flow-cytometry is a new alternative for other commonly used methods, such as optical density measurement or standard plate count, which allows the differentiation of yeast cell populations. Our results showed that there is a positive correlation between the number of CA cells and the hyperglycemic environment, thus providing further evidence on the high incidence of CA infections in patients with poor glucose management. Additionally, we showed that fructose increases the generation time of CA, thus inhibiting its growth rate. Although there is a proven interdependence between the fructose concentration and CA growth rate, the connection between the values is still open for debate. This study is a strong contribute to the knowledge of the numerous pathways that influence the oral cavity and its microbiota: it also remarks the importance of the multidisciplinary approach to the oral pathologies (Mangini et al. 2006). In fact, the correlations between the systemic diseases and the oral manifestations have many links, actually not fully un- derstood, thus, worthy of further investigations by the scientific community (Santacroce et al. 2008).

\section{AUTHORS' CONTRIBUTION}

AMan, CNC, DP, AIS and AMare conceived the study and performed the data analysis; FS and LS contributed to the statistical analysis and reviewed the manuscript; AMan, FT and AMare drafted the manuscript; AMan, FT, FS, LS and AMare critically revised the manuscript for intellectual content. All authors read and approved the final manuscript.

\section{REFERENCES}

Al-Maskari AY, Al-Maskari MY, Al-Sudairy S. Oral manifestations and complications of diabetes mellitus: a review. Sultan Qaboos Univ Med J. 2011; 11(2): 179-86.

Balan P, Gogineni SB, Kumari NS, Shetty V, Rangare AL, Castelino $\mathrm{R}$, et al. Candida carriage rate and growth characteristics of saliva in diabetes mellitus patients: a case-control study. J Dent Res Dent Clin Dent Prospects. 2015; 9(4): 274-9.

Biesta-Peters EG, Reij MW, Joosten H, Gorris LGM, Zwietering MH. Comparison of two optical-density-based methods and a plate count method for estimation of growth parameters of bacillus cereus. Appl Environ Microbiol. 2010; 76(5): 1399-405.

Casqueiro J, Casqueiro J, Alves C. Infections in patients with diabetes mellitus: a review of pathogenesis. Indian J Endocrinol Metab. 2012; Suppl. 1: S27-36.

Cozma AI, Sievenpiper JL, de Souza RJ, Chiavaroli L, Ha V, Wang DD, et al. Effect of fructose on glycemic control in diabetes: a systematic review and meta-analysis of controlled feeding trials. Diabetes Care. 2012; 35(7): 1611-20.

DiNicolantonio JJ, O'Keefe JH, Lucan SC. Added fructose: a principal driver of type 2 diabetes mellitus and its consequences. Mayo Clin Proc. 2015; 90(3): 372-81.

Ene IV, Adya AK, Wehmeier S, Brand AC, MacCallum DM, Gow NA, et al. Host carbon sources modulate cell wall architecture, drug resistance and virulence in a fungal pathogen. Cell Microbiol. 2012; 14(9): 1319-35.

Foladori P, Bruni L, Tamburini S, Ziglio G. Direct quantification of bacterial biomass in influent, effluent and activated sludge of wastewater treatment plants by using flow cytometry. Water Res. 2010; 44(13): 3807-18.

Giudice G, Cutrignelli DA, Spportelli P, Limongelli L, Di Gioia G, Santacroce L, et al. Rhinocerebral zygomycosis with orosinusal involvement: diagnostic and surgical treatment guidelines. Endocr Metab Immune Disord Drug Targets. 2016; 16(4): 264-9.

Gregg EW, Sattar N, Ali MK. The changing face of diabetes complications. Lancet Diabetes Endocrinol. 2016; 4(6): 537-47.

Inchingolo F, Tatullo M, Abenavoli FM, Marrelli M, Inchingolo AD, Palladino A, et al. Oral piercing and oral diseases: a short time retrospective study. Int J Med Sci. 2011; 8(8): 649-52.

Jenkinson HF, Douglas LJ. Interactions between Candida species and bacteria in mixed infections. in polimicrobial diseases. 2002. Available from: https://www.ncbi.nlm.nih.gov/books/NBK2486/.

Jurysta C, Bulur N, Oguzhan B, Satman I, Yilmaz TM, Malaisse WJ, et al. Salivary glucose concentration and excretion in normal and diabetic subjects. J Biomed Biotechnol. 2009; 2009: 6 pp.

Kumar S, Padmashree S, Jayalekshmi R. Correlation of salivary glucose, blood glucose and oral candidal carriage in the saliva of type 2 diabetics: a case-control study. Contemp Clin Dent. 2014; 5(3): 312-7.

Lee D-H, Kim S-J, Seo J-H. Molecular cloning and characterization of two novel fructose-specific transporters from the osmotolerant 
and fructophilic yeast Candida magnoliae JH110. Appl Microbiol Biotechnol. 2014; 98(8): 3569-78.

Mangini F, Santacroce L, Bottalico L. Periodontitis and systemic diseases. Clin Ter. 2006; 157(6): 541-8.

Marrelli M, Tatullo M, Dipalma G, Inchingolo F. Oral infection by Staphylococcus aureus in patients affected by White Sponge Nevus: a description of two cases occurred in the same family. Int $\mathbf{J}$ Med Sci. 2012; 9(1): 47-50.

Mayer FL, Wilson D, Hube B. Candida albicans pathogenicity mechanisms. Virulence. 2013; 4(2): 119-28.

Minea B, Nastasa V, Moraru RF, Kolecka A, Flonta MM, Marincu I, et al. Species distribution and susceptibility profile to fluconazole, voriconazole and MXP-4509 of 551 clinical yeast isolates from a Romanian multi-centre study. Eur J Clin Microbiol Infect Dis. 2015 ; 34(2): 367-83.

Monea A, Santacroce L, Marrelli M, Man A. Oral candidiasis and inflammatory response: a potential synergic contribution to the onset of type-2 diabetes mellitus. AMJ. 2017; 10(6): 550-6.

Nirmala S, Quadar M, Veluru S. pH modulation and salivary sugar clearance of different chocolates in children: a randomized clinical trial. J Indian Soc Pedod Prev Dent. 2016; 34(1): 10.

Petrovan C, Bereczki-Temistocle DL, Man A. Candida in Oral and maxillo-facial pathology: clinical findings and risk factors. J Interdiscip Med. 2017; 2(Suppl. 1): S36-42.

Sabina J, Brown V. Glucose sensing network in Candida albicans: a sweet spot for fungal morphogenesis. Eukaryot Cell. 2009; 8(9): 1314-20.
Santacroce L, Cagiano R, Carlaio RG, Del Prete R, Bottalico L. Dentistry oral hygiene and endocarditis. Pathophysiology and prophylactic therapy. Recenti Prog Med. 2008; 99(10): 516-21.

Santacroce L, Carlaio RG, Bottalico L. Does it make sense that diabetes is reciprocally associated with periodontal disease? Endocr Metab Immune Disord Drug Targets. 2010; 10(1): 57-70.

Santacroce L, Leone D, Valenzano A, Luperto P, Bottalico L, Losacco T. Nutritional problems in the surgical patients with head and neck tumours. Literature review and personal experience. Clin Ter. 2005; 156(5): 227-30.

Satish BNVS, Srikala P, Maharudrappa B, Awanti SM, Kumar P, Hugar D. Saliva: a tool in assessing glucose levels in diabetes mellitus. J Int Oral Health. 2014; 6(2): 114-7.

Scridon A, Perian M, Marginean A, Fisca C, Vantu A, Ghertescu D, et al. Wistar rats with long-term streptozotocin-induced type 1 diabetes mellitus replicate the most relevant clinical, biochemical, and hematologic features of human diabetes. Rev Romana Med Lab. 2015; 23(3): 263-74.

Soysa NS, Samaranayake LP, Ellepola ANB. Diabetes mellitus as a contributory factor in oral candidosis. Diabet Med. 2006; 23(5): 455-9.

Tamayo T, Rosenbauer J, Wild SH, Spijkerman AMW, Baan C, Forouhi NG, et al. Diabetes in Europe: an update. Diabetes Res Clin Pract. 2014; 103(2): 206-17.

Tappy L, Lê K-A. Health effects of fructose and fructose-containing caloric sweeteners: where do we stand 10 years after the initial whistle blowings? Curr Diab Rep. 2015; 15(8): 54.

Vidotto V, Sinicco A, Accattatis G, Aoki S. Influence of fructose on Candida albicans germ tube production. Mycopathologia. 1996; 135(2): 85-8. 